published in

Wave Motion 97, 102612 (2020)

https://doi.org/10.1016/j.wavemoti.2020.102612

\title{
Are waves with negative spatial damping unstable?
}

\author{
Henning Struchtrup* \& Ben Nadler ${ }^{\dagger}$ \\ Department of Mechanical Engineering \\ University of Victoria, Victoria, BC, 8W 2Y2, Canada
}

May 25, 2020

\begin{abstract}
Conventional plane harmonic waves decay in direction of propagation, but unconventional harmonic waves grow in the direction of propagation. While a single unconventional wave cannot be a solution to a physically meaningful boundary value problem, these waves may have an essential contribution to the overall solution of a problem as long as this is a superposition of unconventional and conventional waves. A fourth order diffusion equation with proper thermodynamic structure, and the Burnett equations of rarefied gas dynamics exhibit conventional and unconventional waves. Steady state oscillating boundary value problems are considered to discuss the interplay of conventional and unconventional waves. Results show that as long as the second law of thermodynamics is valid, unconventional waves may contribute to the overall solution, which, however is dominated by conventional waves, and behaves as these.
\end{abstract}

\section{Introduction}

Any mathematical model is expected to fulfill stability requirements, so that small disturbances do not blow up. The amplitude of a small fluctuation in space should not grow over time (temporal stability), and a small disturbance at a boundary should not induce a large disturbance away from the wall (spatial stability). Hence, new models should be tested for stability of both types. The simplest test is for linear stability, where one considers only equations linearized about a homogeneous equilibrium rest state, for small disturbances. If a model is linearly unstable, also the full non-linear model will suffer from instabilities, which might render the model useless.

A well-known example are the Burnett equations of rarefied gas dynamics $[1,2,3]$, which exhibit linear temporal instabilities for disturbances of small wavelengths $[4,5,6]$. Solutions of the fully non-linear Burnett equations for the shock wave structure problem are strongly affected by these linear instabilities. If a numerical time stepping method is used, unavoidable numerical errors of small wavelengths occur on refined grids, which blow up over time, and make the solution impossible [7, 8], or at least introduce artificial oscillations [9].

The (linearized) Burnett equations also exhibit wave solutions with increasing amplitude in direction of propagation, which indicates possible spatial instabilities. The following discussion aims to clarify the relation between such wave modes, and spatial instability.

A plane harmonic wave of frequency $\omega>0$ and wavelength $\lambda=\frac{2 \pi}{|k|}$ is given by the equation $[10,11,3]$

$$
u(x, t)=u_{0} e^{-\zeta x} \cos [\omega t-k x+\phi],
$$

*e-mail: struchtr@uvic.ca

${ }_{\dagger}^{\dagger}$-mail: bnadler@uvic.ca 
where $u_{0}$ is the amplitude, $k$ is the (real) wave number, $\phi$ is the phase offset, and the spatial damping coefficient $\zeta$ describes the change of amplitude in space. The wave travels with the phase speed $v_{p h}=\omega / k$ into positive $x$-direction for $k>0$ and into negative $x$-direction for $k<0$.

On physical grounds, for an individual wave that emerges from a source at $x=0$, one would expect positive spatial damping, such that the amplitude decreases in the direction of travel, i.e., $\zeta>0$ if $k>0$ and $\zeta<0$ if $k<0[11,3]$. For instance, the classical heat equation leads to wave solutions with $k=\zeta= \pm \sqrt{\omega / 2}$, hence behaves as expected. The same is true for the description of damped sound waves by means of the linearized Navier-Stokes equations.

There are, however, models in the literature, where the solution includes waves with negative spatial damping, such that the amplitude grows in the direction of propagation, i.e., $\pm \zeta<0$ for $\pm k>0$. Well-known examples arise in continuum models based on rarefied gas dynamics [2,3], which extend the Navier-Stokes and Fourier equations by adding higher order terms: The Burnett [1] and super-Burnett [12] equations, and some related models, such as the augmented Burnett equations [13], exhibit wave modes of this unconventional type $[6,3]$.

In previous work, it was stated that these unconventional waves should not appear in solutions of transport models, so that models that exhibit such "spatially unstable" waves should be discarded $[6,3,11]$. However, these statements were not corroborated by performing actual solutions of the incriminated equations for boundary value problems.

In the following we will show that, in contrast to what was stated earlier $[6,3,11]$, unconventional contribute to meaningful solutions of proper thermodynamic models. The overall solutions are superpositions of several conventional and unconventional waves, and these behave properly, when unconventional waves contribute, but conventional waves dominate the solution. We consider two test cases, the diffusion equation with an additional fourth order term and the linearized Burnett equations. The results indicate that the superpositions are meaningful when they solve equations that are accompanied by a proper formulation of the second law of thermodynamics.

The fourth order diffusion equation is constructed based on the principles of non-equilibrium thermodynamics [14] such that it is accompanied by a proper statement of the second law of thermodynamics, that it has an entropy with positive production term. The entropy flux is used to construct entropy generating boundary conditions. The equation is solved for the oscillating steady state with an oscillating boundary. It will be seen that although unconventional waves contribute significantly, the overall result behaves as expected, with a signal leaving the boundary with decreasing amplitude in the direction of propagation.

The Burnett equations [1] are well known to be unstable [4] and violating the second law of thermodynamics [15], at least for large frequencies [16]. We consider the equations for the setting of an acoustic resonator [17], i.e., a boundary value problem. At small frequency the equations describe sound waves leaving the oscillating boundary with decreasing amplitude in the direction of propagation. For larger frequency, however, the unconventional modes are dominant, and the equations produce a nonsensical result, namely waves arriving at the oscillating boundary, with increasing amplitude in the direction of propagation. In this case, the oscillator produces power - a clear violation of the second law.

We are not aware of discussion of unconventional wave modes by others. Hence, this contribution aims to clarify the relevance of such waves. The results indicate that these waves might contribute to reasonable results, as long as the underlying equations do not violate the second law of thermodynamics. Occurrence of these waves does not justify to discard a set of transport equations. However, one has to carefully examine the equations, since unconventional waves, if dominant, lead to nonsensical results. 


\section{Plane Harmonic Waves}

\subsection{Dispersion Relation}

We consider plane harmonic waves in complex formulation as solution of partial differential equations [10,11]. A set of $n$ variables $u_{A}(x, t), A=1, \ldots, n$ obeys a set of linear partial differential equations of the form

$$
\frac{\partial u_{A}}{\partial t}+A_{A B}^{(1)} \frac{\partial u_{B}}{\partial x}+A_{A B}^{(2)} \frac{\partial^{2} u_{B}}{\partial x^{2}}+\cdots+A_{A B}^{(n)} \frac{\partial^{n} u_{B}}{\partial x^{n}}=-C_{A B} u_{B}
$$

with constant coefficient matrices $A_{A B}^{(j)}, C_{A B}$. Plane harmonic wave are a class of solutions for such a system, where all variables are assumed to be of the complex form

$$
u_{A}=\hat{u}_{A} \exp [i(\omega t-k x)] .
$$

Here, $\omega$ denotes frequency, $k$ denotes wave number, and $\hat{u}_{A}$ denotes complex amplitude. While only the real part of $u_{A}$ is relevant, it is advantageous to perform all calculations in the complex form, and take the real part only at the end of calculations.

Insertion of the wave ansatz into the system (2) yields a homogeneous system for the amplitudes $\hat{u}_{A}$,

$$
\left[i \omega \delta_{A B}+(-i k) A_{A B}^{(1)}+(-i k)^{2} A_{A B}^{(2)}+\cdots+(-i k)^{n} A_{A B}^{(n)}+C_{A B}\right] \hat{u}_{B}=\mathcal{A}_{A B}(\omega, k) \hat{u}_{B}=0
$$

Non-trivial solutions require that the determinant of the coefficient matrix vanishes, i.e.,

$$
\operatorname{det}\left[\mathcal{A}_{A B}(\omega, k)\right]=0 .
$$

This relation between frequency $\omega$ and wave number $k$ is known as the dispersion relation. Note that $\operatorname{det}\left[\mathcal{A}_{A B}(\omega, k)\right]$ is a polynomial in both, $k$ and $\omega$.

\subsection{Initial Value Problems: Stability in Time}

The further proceedings depend on the type of problem one wishes to consider. For an initial value problem, one assumes waves of a particular real wave number $k$ (that is of wavelength $\lambda=\frac{2 \pi}{k}$ ) and finds from the dispersion relation a set of $\bar{\nu}$ complex solutions for frequency, $\omega^{\alpha}(k)=\omega_{r}^{\alpha}(k)+i \omega_{i}(k), \alpha=1, \ldots, \bar{\nu}$. The real part $\omega_{r}^{\alpha}$ is directly related to phase velocity $v_{p h}^{\alpha}=\omega_{r}^{\alpha}(k) / k$ and the temporal damping coefficient is given by $\omega_{i}^{\alpha}(k)$, so that the wave solution for mode $\alpha$ can be written as

$$
u_{A}^{\alpha}(x, t ; k)=\hat{u}_{A}^{\alpha}(k) e^{-\omega_{i}^{\alpha}(k) t} \exp \left[i k\left(v_{p h}^{\alpha}(k) t-x\right)\right],
$$

where $\hat{u}_{A}^{\alpha}(k)$ is a complex amplitude.

Meaningful transport equations require stability in time, in the sense that the amplitude of an initial disturbance should not grow over time, but either stay constant or decay. Hence, for all modes, linear stability is ensured for

$$
\omega_{i}^{\alpha}(k) \geq 0 .
$$

All typical transport equations, such as wave and diffusion equations, the Navier-Stokes equations, etc., are stable in this sense. Stability tests are highly useful in modelling, where this is one of the first consistency tests one will perform [6]. Typically, instability is linked to violation of the second law of thermodynamics.

A well-known example for an unstable model are the Burnett equations of rarefied gas dynamics [1], for which waves of larger wavelengths are stable, but those with shorter wavelength are unstable [4]. The instability can be linked to the loss of a proper second law for the equations [15]. The instability renders the 
equations effectively useless for numerical simulations, since unavoidable numerical errors on fine grids will induce waves of small wavelength, which will blow up over time exponentially $[7,8,9]$.

For stable equations, solutions of initial value problems are obtained from superposition of all modes at all wavelengths, $u_{A}(x, t)=\int \sum_{\alpha} u_{A}^{\alpha}(x, t ; k) d k$, where the amplitudes $\hat{u}_{A}^{\alpha}(k)$ are obtained from the initial conditions. Typically, this approach to solution of initial value problems requires solutions in the infinite space domain. Certain boundary values problems might be accessible through clever continuation of initial values from the bounded domain to the infinite domain, but in general, i.e., for systems with more complicated boundary conditions such as those discussed below, this will not be possible.

\subsection{Boundary Value Problems: Conventional and Unconventional Waves}

For a boundary value problem, one prescribes oscillations of real frequency $\omega>0$ at a location (say, $x=0$ ), and finds from the dispersion relation a set of $\nu$ complex solutions for wave number, $k^{\alpha}(\omega)=k_{r}^{\alpha}(\omega)+i k_{i}^{\alpha}$, $\alpha=1, \ldots, \nu$. The real part is directly related to phase velocity as $v_{p h}(\omega)=\omega / k_{r}^{\alpha}(\omega)$, so that the wave solution for mode $\alpha$ reads

$$
u_{A}^{\alpha}(x, t ; \omega)=\hat{u}_{A}^{\alpha}(\omega) e^{k_{i}^{\alpha}(\omega) x} \exp \left[i \omega\left(t-\frac{x}{v_{p h}^{\alpha}(\omega)}\right)\right]
$$

For positive phase velocities, a wave propagates towards positive $x$ (i.e., to the right), while for negative phase velocity, the wave travels towards negative $x$ (i.e., to the left). For typical transport equations (wave, diffusion, etc.) with positive spatial damping, the amplitude of a wave decays in the direction of travel, so that

$$
k_{i}^{\alpha}(\omega)<0 \quad \text { if } \quad v_{p h}^{\alpha}(\omega)>0, \quad \text { and } \quad k_{i}^{\alpha}(\omega)>0 \quad \text { if } \quad v_{p h}^{\alpha}(\omega)<0 \quad \text { (conventional). }
$$

However, there are (systems of) equations that have at least some modes with negative spatial damping, for which the signs are opposite, so that

$$
k_{i}^{\alpha}(\omega)>0 \quad \text { if } \quad v_{p h}^{\alpha}(\omega)>0, \quad \text { and } \quad k_{i}^{\alpha}(\omega)<0 \quad \text { if } \quad v_{p h}^{\alpha}(\omega)<0 \quad \text { (unconventional). }
$$

Accordingly, the amplitude of these modes grows in direction of propagation. For instance, such modes occur in higher order models in rarefied gas dynamics, such as Burnett-type models. In previous work such modes were described as being "unstable in space", and considered to be unphysical, so that equations that have such modes should be discarded [6,3]. As the discussion below will show, these modes might be problematic, but their occurrence does not suffice to judge on a transport model. Therefore, as already in the introduction, we shall refer to "conventional", and "unconventional" modes, and not refer to "spatial stability."

The general solution of a boundary value problem is a superposition of all modes at all frequencies of the form $u_{A}(x, t)=\int \sum_{\alpha} u_{A}^{\alpha}(x, t ; \omega) d \omega$, where the amplitudes $\hat{u}_{A}^{\alpha}(\omega)$ are obtained from boundary conditions. Typically, in this approach it is not posssible to prescribe initial conditions.

Two transport models that produce unconventional waves will be studied below, an extended diffusion model with proper second law structure, and the Burnett equations of rarefied gas dynamics which are well known to violate the second law.

\section{Extended Diffusion Equation}

\subsection{Equation and Boundary Conditions}

Loss of stability in time is typically linked to a breakdown of the second law of thermodynamics, but unconventional waves may occur in entropic models. Here, we use as an example an extended diffusion equation, which is accompanied by a proper formulation of the second law. 
The model is based on an entropy with non-equilibrium contributions, for which one can find a balance equation with positive entropy generation. The constitutive equations for the energy flux and the boundary conditions are deduced from the second law, such that all processes in the bulk and at the boundaries are dissipative. The model can also be derived from a simple kinetic model, that is it is rooted in meaningful physics. Details of this procedure are presented in the Appendix, here we only summarize the resulting transport equation and boundary conditions for the case of interest.

The higher order diffusion equation for the deviation of energy (or temperature) from a homogenous groundstate $u(x, t)$ reads

$$
\frac{\partial u}{\partial t}-\frac{\partial^{2} u}{\partial x^{2}}+\xi \frac{\partial^{4} u}{\partial x^{4}}=0
$$

where $\xi>0$ is a constant. We shall consider problems where at the left boundary, at $x=0$, the boundary energy $u_{B}(t)=u_{0} \cos \omega t$ is periodic, with the jump boundary conditions (40),

$$
\begin{aligned}
& -\frac{\partial u}{\partial x \mid 0}+\left(\tau_{1}+\tau_{2}\right) u_{\mid 0}-\tau_{1} \xi^{2} \frac{\partial^{4} u}{\partial x^{4}}-\left.\tau_{2} \xi \frac{\partial^{2} u}{\partial x^{2}}\right|_{0}=\left(\tau_{1}+\tau_{2}\right) u_{B}(t) \\
& \xi \frac{\partial^{3} u}{\partial x^{3}{ }_{\mid 0}}+\left(\tau_{2}+\tau_{3}\right) u_{\mid 0}-\tau_{2} \xi^{2} \frac{\partial^{4} u}{\partial x^{4}}-\left.\tau_{3} \xi \frac{\partial^{2} u}{\partial x^{2}}\right|_{0}=\left(\tau_{2}+\tau_{3}\right) u_{B}(t)
\end{aligned}
$$

The right boundary is either infinitely far away (semi-infinite problem), or an adiabatic boundary at $x=L$, for which (40) reduce to

$$
\frac{\partial u}{\partial x} \mid L_{\mid L}=-\xi{\frac{\partial^{3} u}{\partial x^{3}} \mid L}=0
$$

The constant coefficients can be chosen freely, as long as $\xi \geq 0, \tau_{1} \geq 0,\left(\tau_{1} \tau_{3}-\tau_{2}^{2}\right) \geq 0$. For all computations below we set $u_{0}=1, \xi=1, \tau_{1}=\tau_{3}=1, \tau_{2}=\frac{1}{4}$.

The classical diffusion equation and its jump boundary conditions are obtained by setting $\xi=\tau_{2}=\tau_{3}=$ 0 .

\subsection{Dispersion Relation and Modes}

Insertion of the wave ansatz (3) into the diffusion equation (11) yields the dispersion relation

$$
i \omega+k^{2}+\xi k^{4}=0 .
$$

For an initial value problem, where $k$ is real, the (single) purely complex frequency is

$$
\omega=i \omega_{i}=i k^{2}\left(1+\xi k^{2}\right) .
$$

Convexity of the entropy (29) requires $\xi>0$. Accordingly, the damping $\omega_{i}(k)$ is always positive, and waves of any wavelength are stable in time. In other words, with the second law guaranteed, the extended diffusion model is unconditionally stable.

We note that violation of the second law occurs for $\xi<0$. In that case waves with small wavelengths, so that $k^{2}>1 /|\xi|$, become unstable: instability in time is linked to loss of entropic structure. We proceed with the entropic equation, that is consider $\xi>0$ only.

For boundary value problems, where $\omega$ is real, we find four complex modes,

$$
k_{1,2}= \pm \frac{1}{\sqrt{2 \xi}} \sqrt{-1+\sqrt{1-i 4 \xi \omega}} \quad, \quad k_{3,4}= \pm \frac{1}{\sqrt{2 \xi}} \sqrt{-1-\sqrt{1-i 4 \xi \omega}} .
$$

Figure 1 shows the four modes in the complex plane with $\omega$ as curve parameter. Modes $k_{1}, k_{2}$ are conventional modes, while $k_{3}, k_{4}$ are unconventional modes, for which the amplitude grows in the direction of propagation. 


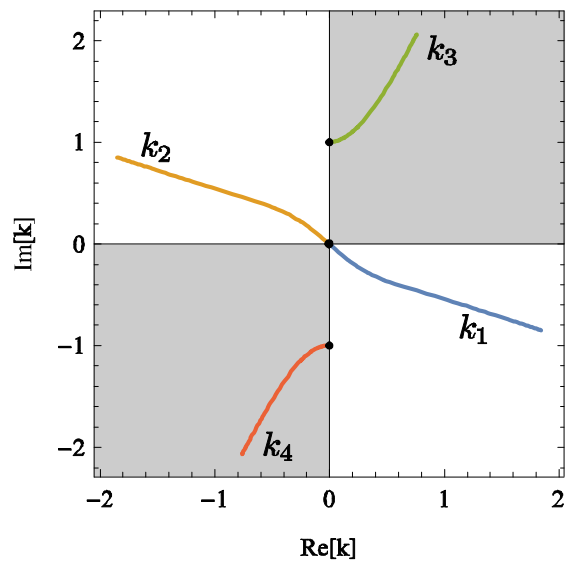

Figure 1: The solutions $k(\omega)$ of the dispersion relation in the complex plane with $\omega$ as curve parameter. Modes $k_{1}, k_{2}$ are conventional modes, while modes $k_{3}, k_{4}$ are unconventional. The dots denote the points where $\omega=0$.

\subsection{Semi-infinite Oscillating Steady State}

We consider problems in which all boundary disturbances are periodic with a single frequency $\omega$. The general solution of the transport equation is a superposition of the four modes,

$$
u(x, t)=\operatorname{Re}\left[\sum_{\alpha=1}^{4} \hat{u}_{\alpha} \exp \left[i\left(\omega t-k_{\alpha} x\right)\right]\right] .
$$

Here, $\hat{u}_{\alpha}$ are the complex amplitudes of the four modes which must be determined from boundary conditions

The apparent problem with unconventional waves becomes most visible when one considers a semi-infinite problem, where a periodic boundary disturbance is created at $x=0$, with the boundary condition (12) and $u_{B}=u_{0} \cos \omega t$. Considering that the disturbance originates at $x=0$, and looking at transport towards $x>0$, one might be inclined to assume that only waves with positive wave speeds play a role, which are induced at the boundary, and travel into the domain. With this assumption, only modes with $\operatorname{Re}\left[k_{\alpha}\right]>0$ contribute, that is conventional mode \#1 and unconventional mode \#3. Since mode \#3 grows in the direction of propagation $\left(\operatorname{Im}\left[k_{3}\right]>0\right.$, see Fig. 1 ) this leads to a nonsensical result, where the energy $u(x, t)$ blows up away from the oscillating boundary.

Indeed, for this problem, one will expect a solution that decays away from the source at $x=0$, and based on this, only modes with $\operatorname{Im}\left[k_{\alpha}\right]<0$ can contribute, i.e., modes \#1 and \#4, so that

$$
u(x, t)=\operatorname{Re}\left[\hat{u}_{1} \exp \left[i\left(\omega t-k_{1} x\right)\right]+\hat{u}_{4} \exp \left[i\left(\omega t-k_{4} x\right)\right]\right] .
$$

While mode \#4 appears to travel to the left, i.e., to come "out of infinity", this result is sensible, as will be explained now. Indeed, what matters is not the individual modes, but only the superposition of all modes. That is, in the present case, the overall signal $u(x, t)$ must travel from the source towards $x>0$ with decaying amplitude. Individual waves are mathematical constructs, that have no meaning for observed transport as long as the overall signal is a superposition.

To evaluate the propagation of the oscillating overall signal $u(x, t)$ one might for instance consider the velocity of axis intercepts. If $x_{n}(t)$ solves $u\left(x_{n}(t), t\right)=0$, the speed of signal propagation is

$$
v_{S}=\frac{d x_{n}}{d t}=-\frac{\left(\frac{\partial u}{\partial t}\right)_{\mid x_{n}}}{\left(\frac{\partial u}{\partial x}\right)_{\mid x_{n}}}
$$


It is clear from Eqs. $(18,19)$ that the signal speed depends strongly on the relative weight of the two modes as expressed by the complex amplitudes $\hat{u}_{1}$ and $\hat{u}_{4}$, which must be determined from the boundary conditions (12). We have found positive signal velocities for all frequencies. That is, for the extended diffusion model, the unconventional wave contributes to the overall signal, but the overall signal propagates into the same direction as the conventional wave. Curves of $u(x, t)$ for the semi-infinite domain agree with those shown below for finite domain, hence are not shown.

\subsection{Finite Oscillating Steady State}

For further insight, we present some figures with results for a finite domain. We consider the transport equation (11) with the boundary conditions $(12,13)$, with oscillating boundary energy $u_{B \mid 0}(t)=u_{0} \cos \omega t$, where $u_{0}=1, \omega=1$. The amplitudes $\hat{u}_{\alpha}$ are obtained from the boundary conditions at $x=0$ and $x=L$, as

$$
\hat{u}_{\beta}=A_{\alpha \beta}^{-1}\left(\begin{array}{c}
\left(\tau_{1}+\tau_{2}\right) u_{0} \\
\left(\tau_{1}+\tau_{2}\right) u_{0} \\
0 \\
0
\end{array}\right)_{\beta}
$$

where $A_{\alpha \beta}^{-1}$ is the inverse of a matrix $A_{\alpha \beta}$ with the elements

$$
\begin{aligned}
& A_{1 \beta}=\left(\tau_{1}+\tau_{2}\right)+i k_{\beta}-\tau_{1} \xi^{2} k_{\beta}^{4}+\tau_{2} \xi k_{\beta}^{2}, \\
& A_{2 \beta}=\left(\tau_{2}+\tau_{3}\right)+i \xi k_{\beta}^{3}-\tau_{2} \xi^{2} k_{\beta}^{4}+\tau_{3} \xi k_{\beta}^{2} \\
& A_{3 \beta}=\left[i k_{\beta}\right] \exp \left[-i k_{\beta} L\right] \quad, \quad A_{4 \beta}=\left[i \xi k_{\beta}^{3}\right] \exp \left[-i k_{\beta} L\right]
\end{aligned}
$$

With this, the oscillating solution is readily available without any approximations, and can be examined. Figure 2 shows, for $L=20$, the energy distribution $u(x, t)$ at several times over one half-period of the boundary oscillation. The figure compares the solution of the fourth order diffusion equation (continuous, brown) to that of the classical diffusion equation $(\xi=0$, dashed, blue). Notably, the boundary conditions lead to a jump between boundary and the domain, so that $u(0, t) \neq u_{B \mid 0}(t)$. The fourth order equation leads to differences from classical diffusion in particular close the the left boundary, but the overall behavior of the signal travelling to the right is rather similar. The figures also visualize that the overall signal speed $v_{S}$ as defined in (19) is positive, since the first axis intercept moves to the right.

Figure 3 shows the contributions of the individual modes in comparison to the overall signal (dashed, brown) at time $t=1.49$, where conventional and unconventional modes are shown in blue and red, respectively. In each figure, the $u$-scale is chosen such that one can identify the contribution of each mode; accordingly, subfigures for modes \#2,3,4 do not show the full $u$-curve. A closer look at the results shows that the adiabatic boundary conditions on the right lead to a reflection of the waves, such that $u_{1}(L, t)=u_{2}(L, t)$ and $u_{3}(L, t)=u_{4}(L, t)$; this is not visible in the figures due to lack of resolution.

For interpretation, we recall that the oscillation is initiated at the left boundary, while the right boundary is adiabatic. The main contribution to the overall signal comes from conventional mode \#1, which emanates from the left boundary and travels to the right, where it is reflected into mode \#2. Unconventional mode \#4, which emanates from the right as the reflection of mode \#3, travels to the left and contributes mostly in the vicinity of the left boundary, due to the unconventional exponential increase of amplitude in direction of propagation. One can say that this mode contributes a boundary layer at the left, but its contribution does not lead to an inversion of the signal speed. 

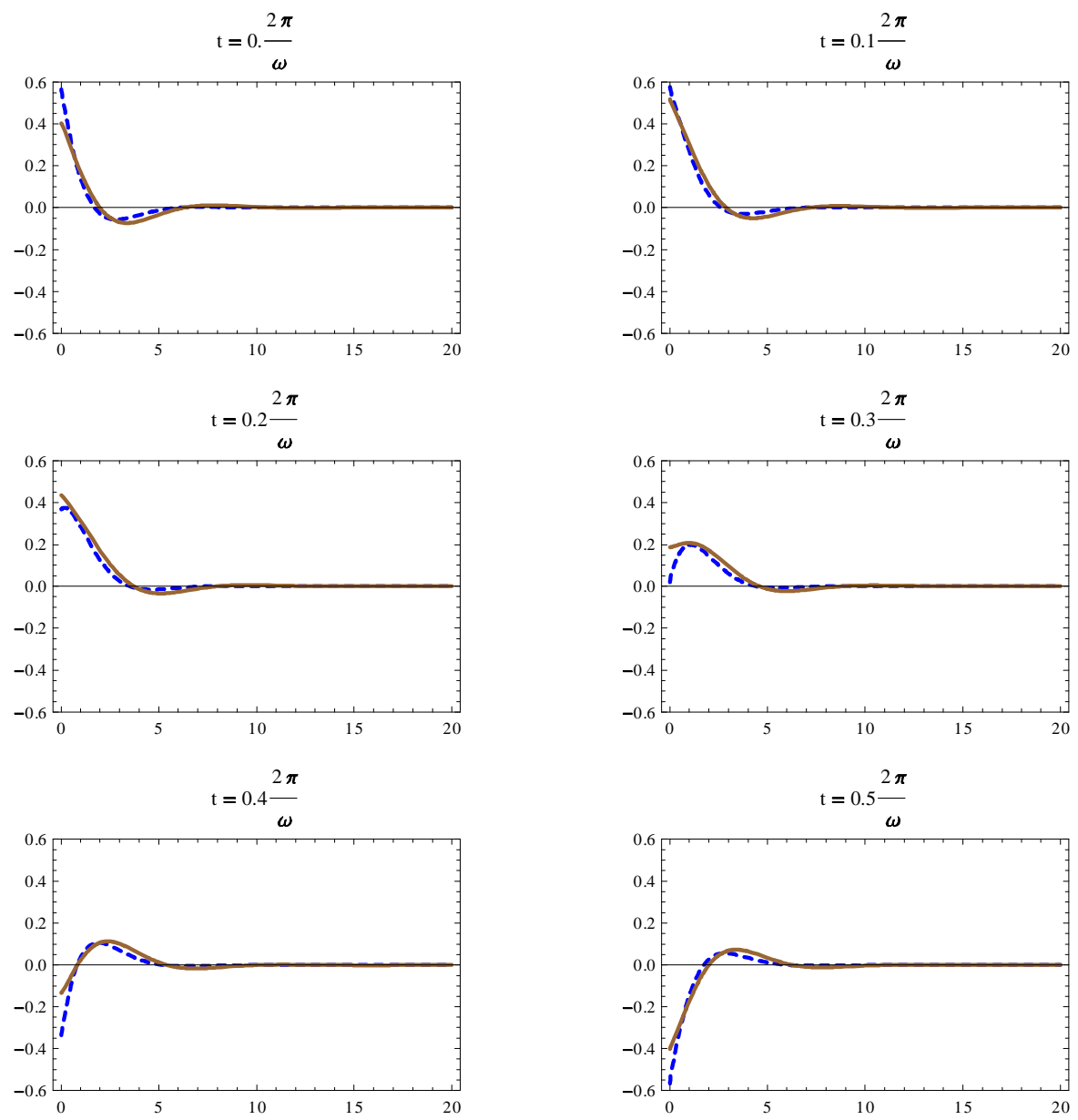

Figure 2: $u(x, t)$ for $\omega=1, L=20$ over one half-period of the boundary oscillation: solution of 4 th order equation (continuous, brown) compared to solution of classical diffusion equation (dashed, blue). 

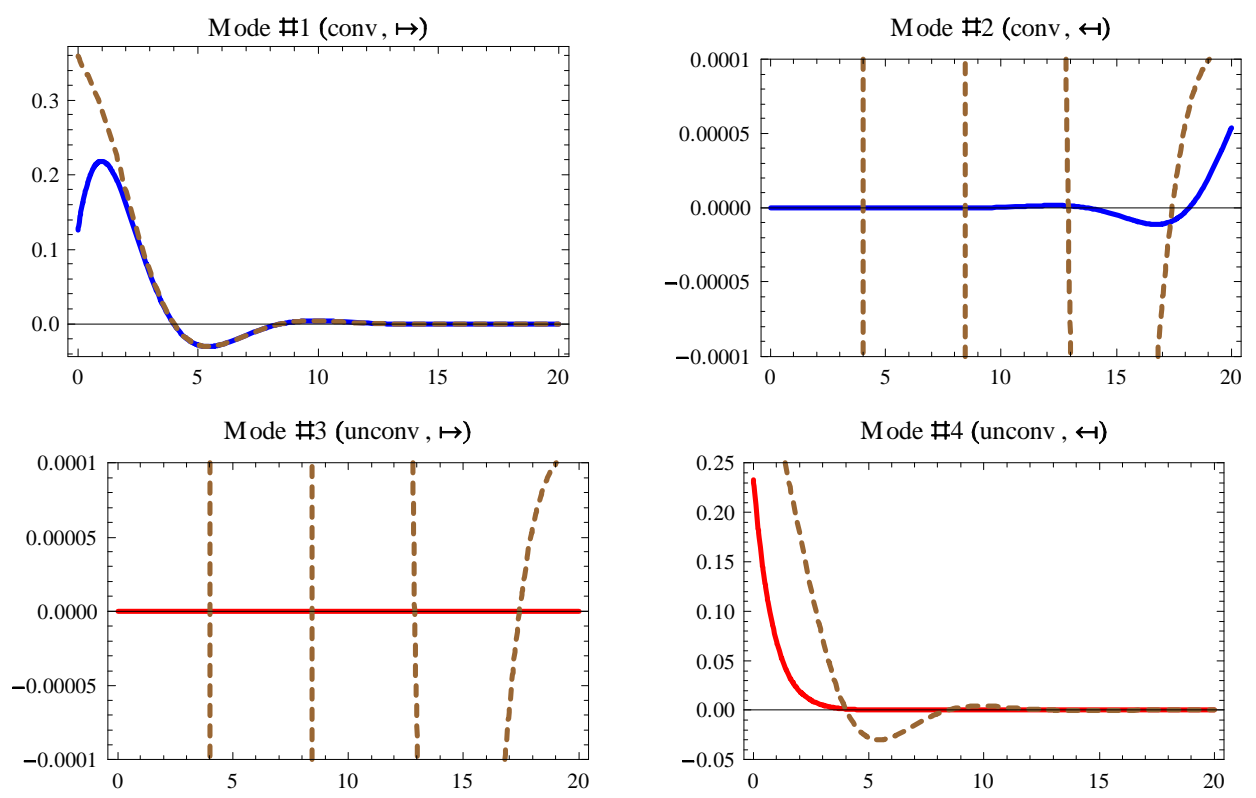

Figure 3: Results for $\omega=1, L=20$ at $t=1.49$. Overall energy $u(x, t)$ (dashed, orange) in overlay with the individual contributions of the four modes. First row: conventional modes (continuous, blue); second row: unconventional modes (continuous, red). Left column: Waves travelling to the right $(\rightarrow$ ); right column: waves travelling to the left $(\leftarrow)$.

\subsection{Loss of Entropy}

The extended diffusion equation and its boundary conditions are constructed to ensure validity of the second law. Choosing negative values for the coefficient $\xi$ gives a model for which the second law is not satisfied, with temporal instability for initial value problems at small wavelength, i.e., $k>1 / \sqrt{|\xi|}$, see the discussion below Eq. (15). Also in this case one finds one pair of conventional and one pair of unconventional waves. Calculations for the above boundary value problem, but now with negative coefficient $\xi=-1$, give results $u(t, x)$ that are dominated by the left-going unconventional wave, with, indeed, $v_{S}<0$ for the overall signal. This behavior is somewhat similar to that of the Burnett equations that we discuss next, hence we do not provide additional details or figures. From this observation one might conjecture that it is not the unconventional waves that are the problem, but rather the loss of the second law.

For further insight, we also performed numerical solutions for initial-boundary value problems with initial conditions $u(x, t=0)=0$. For the entropic model $(\xi>0)$ these agree with the steady state oscillations very soon, after about one period of the boundary signal $u_{B}(t)$, independent of domain length $L$. For the non-entropic model $(\xi<0)$, the temporal instability makes it impossible to produce meaningful results, since the discretization introduces numerical errors at small wavelengths which grow exponentially in time.

\section{Burnett Equations}

\subsection{Equations and Boundary Conditions}

The Burnett equations are derived from the Boltzmann equation as a model for slightly rarefied flows of ideal gases $[1,2,3]$. We consider the equations in linearized dimensionless form, where all variables indicate 
dimensionless deviation from a homogeneous rest state. The variables are mass density $\rho$, flow velocity $v$, temperature $\theta$, with constitutive relations for non-equilibrium stress $\sigma$ and heat flux $q$. From the ideal gas law, the pressure deviation is $p=\rho+\theta$. For 1-dimensional problems, the equations read

$$
\begin{aligned}
\frac{\partial \rho}{\partial t}+\frac{\partial v}{\partial x} & =0 \\
\frac{\partial v}{\partial t}+\frac{\partial \rho}{\partial x}+\frac{\partial \theta}{\partial x}+\frac{\partial \sigma}{\partial x} & =0 \\
\frac{3}{2} \frac{\partial \theta}{\partial t}+\frac{\partial q}{\partial x}+\frac{\partial v}{\partial x} & =0 \\
{\left[\frac{4}{3} \frac{\partial^{2} \rho}{\partial x^{2}}-\frac{2}{3} \frac{\partial^{2} \theta}{\partial x^{2}}\right]+\frac{4}{3} \frac{\partial v}{\partial x} } & =-\sigma \\
{\left[\frac{7}{6} \frac{\partial^{2} v}{\partial x^{2}}\right]+\frac{5}{2} \frac{\partial \theta}{\partial x} } & =-\frac{2}{3} q
\end{aligned}
$$

Without the terms in square brackets, these equations are the Navier-Stokes-Fourier equations of classical hydrodynamics (linearized, dimensionless). The additional terms describe higher order effects that are expected for moderately rarefied gases. Specifically, the Burnett equations reduce to the Navier-StokesFourier equations for very small Knudsen numbers, and their validity is restricted to somewhat small Knudsen numbers. For wave problems this requires wavelengths sufficiently larger than the mean free path of the particles, or frequencies sufficiently below the collision frequency. The lengthscale to non-dimensionalize the equations above is of the order of the mean free path, so that frequency or wave number can be interpreted as Knudsen numbers [17]. Hence validity of the above Burnett equations is expected for small $\omega$ or $k$.

It is well known that the Burnett equations are not accompanied by an entropy, that is they violate the 2nd law of thermodynamics. In particular, they exhibit temporal instabilities for small wavelengths, i.e., two of the three frequency modes $\omega_{i}^{\alpha}(k)$ become unstable for dimensionless wave numbers $|k|>2.46$.

For boundary value problems, the Burnett equations have six modes $k_{\alpha}(\omega)$, where two pairs of modes are of conventional type for all frequencies, while one pair of modes changes their type from conventional (for lower frequencies) to unconventional (for frequencies $\omega>5.96$ ). Interestingly, the modes change sign just at wave numbers $\left|k_{r}^{\alpha}\right|=2.46$, corresponding to the wavelength where temporal instability begins. There might to be a relation between the temporal instability and the unconventional mode, but this was not further explored.

The Burnett equations where solved for an acoustic resonator in Ref. [17] with the following jump and slip boundary conditions, motivated from kinetic theory,

$$
\begin{gathered}
v=v_{W}(t) \\
\theta-\frac{1}{3} \frac{\partial v}{\partial x}-\frac{2-\chi}{2 \chi} \sqrt{\frac{\pi}{2}} \frac{15}{4} \frac{\partial \theta}{\partial x} \nu-\frac{1}{3} \frac{\partial^{2} \rho}{\partial x^{2}}+\frac{115}{42} \frac{\partial^{2} \theta}{\partial x^{2}}-\frac{2-\chi}{2 \chi} \sqrt{\frac{\pi}{2}} \frac{7}{4} \frac{\partial^{2} v}{\partial x^{2}} \nu+\frac{6}{5} \frac{\partial^{3} v}{\partial x^{3}}=\theta_{W}(t) \\
\theta+\frac{14}{3} \frac{\partial v}{\partial x}+\frac{14}{3} \frac{\partial^{2} \rho}{\partial x^{2}}-\frac{125}{42} \frac{\partial^{2} \theta}{\partial x^{2}}-8 \frac{2-\chi}{2 \chi} \sqrt{\frac{\pi}{2} \frac{\partial^{2} v}{\partial x^{2}} \nu} \\
-\frac{3}{10} \frac{\partial^{3} v}{\partial x^{3}}-8 \frac{2-\chi}{2 \chi} \sqrt{\frac{\pi}{2}} \frac{\partial^{3} \rho}{\partial x^{3}} \nu+4 \frac{2-\chi}{2 \chi} \sqrt{\frac{\pi}{2}} \frac{\partial^{3} \theta}{\partial x^{3}} \nu=\theta_{W}(t) .
\end{gathered}
$$

Here, $\nu$ denotes the (1-dimensional) wall normal, $v_{W}(t), \theta_{W}(t)$ are the velocity and temperature variations of the wall, and $\chi$ is the wall accommodation coefficient which we set to $\chi=1$. For the resonator, one sets at the left: $x=0, \nu=1, v_{W}(t)=v_{0} \cos \omega t, \theta_{W}=0$, and at the right: $x=0, \nu=-1, v_{W}(t)=\theta_{W}=0$. 

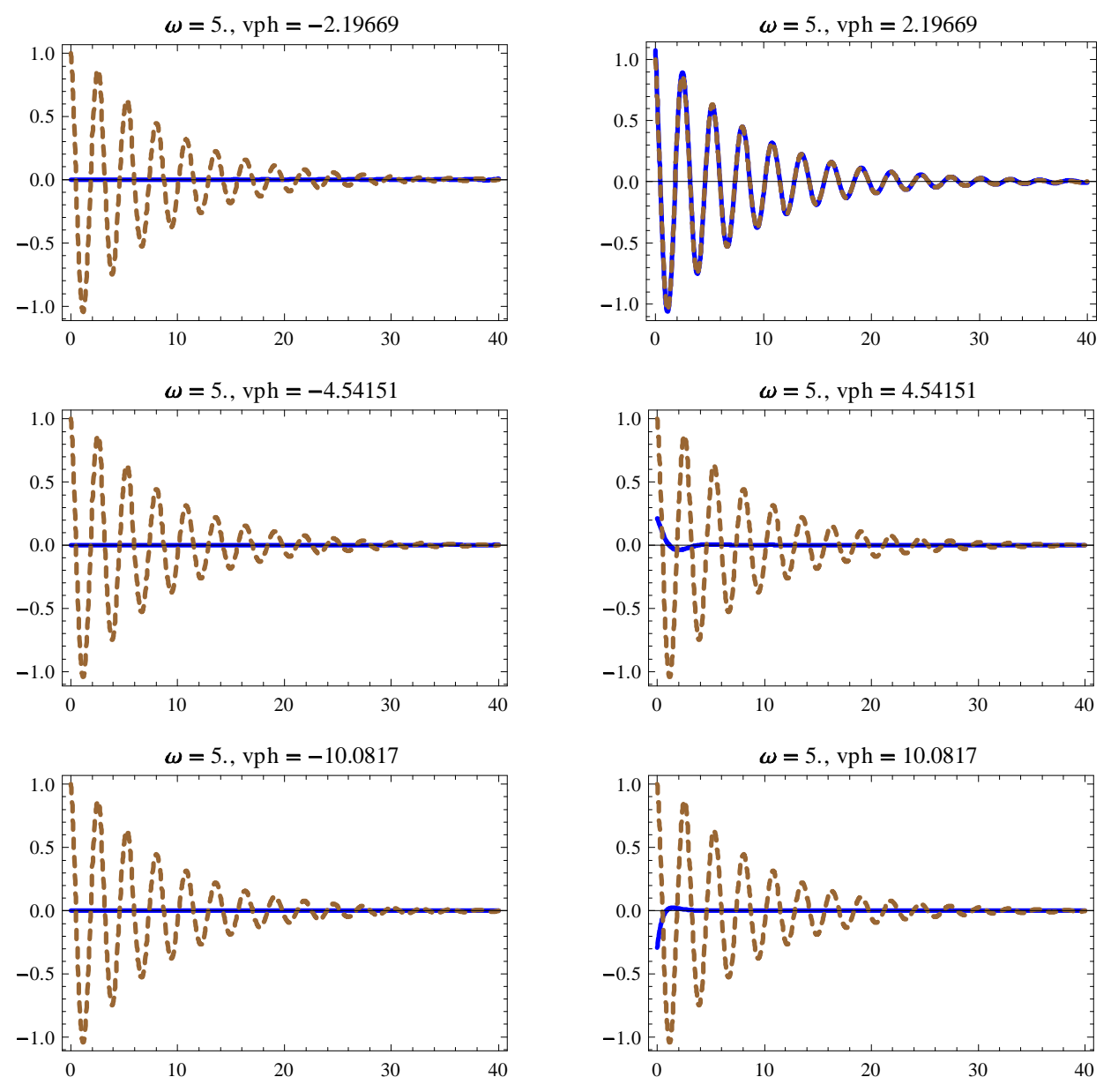

Figure 4: Resonator solution for Burnett equations at $\omega=5, t=0$ : Velocity $v(x, t)$ (dashed, brown) and contributions of the six modes as functions of space. All six modes are conventional (blue).

\subsection{Wave Solutions}

The method of solution for the resonator problem for the Burnett equations is very similar to what we did before, see [17] for more detail. We show two sets of results, for $L=40$, and frequencies $\omega=5$ and $\omega=7$, respectively.

Figure 4 shows, for $\omega=5$, the overall result (dashed, brown) compared to the contributions of the six modes (continuous, blue). All modes are of conventional type, and only modes emanating from the left boundary contribute significantly to the overall signal (figures in right column). The two faster modes contribute only boundary layer effects in the vicinity of the oscillating boundary, while the slowest mode $\left(v_{p h}=2.197\right)$ carries the sound signal into the domain.

The work (per area) to drive the resonator over one period of the boundary signal is the integrated product of velocity and normal force,

$$
W=\int_{0}^{\frac{2 \pi}{\omega}} v(0, t)[p(0, t)+\sigma(0, t)] d t .
$$

For $\omega=5$ the computation gives positive work $W=0.623>0$, which means that work is required to drive 

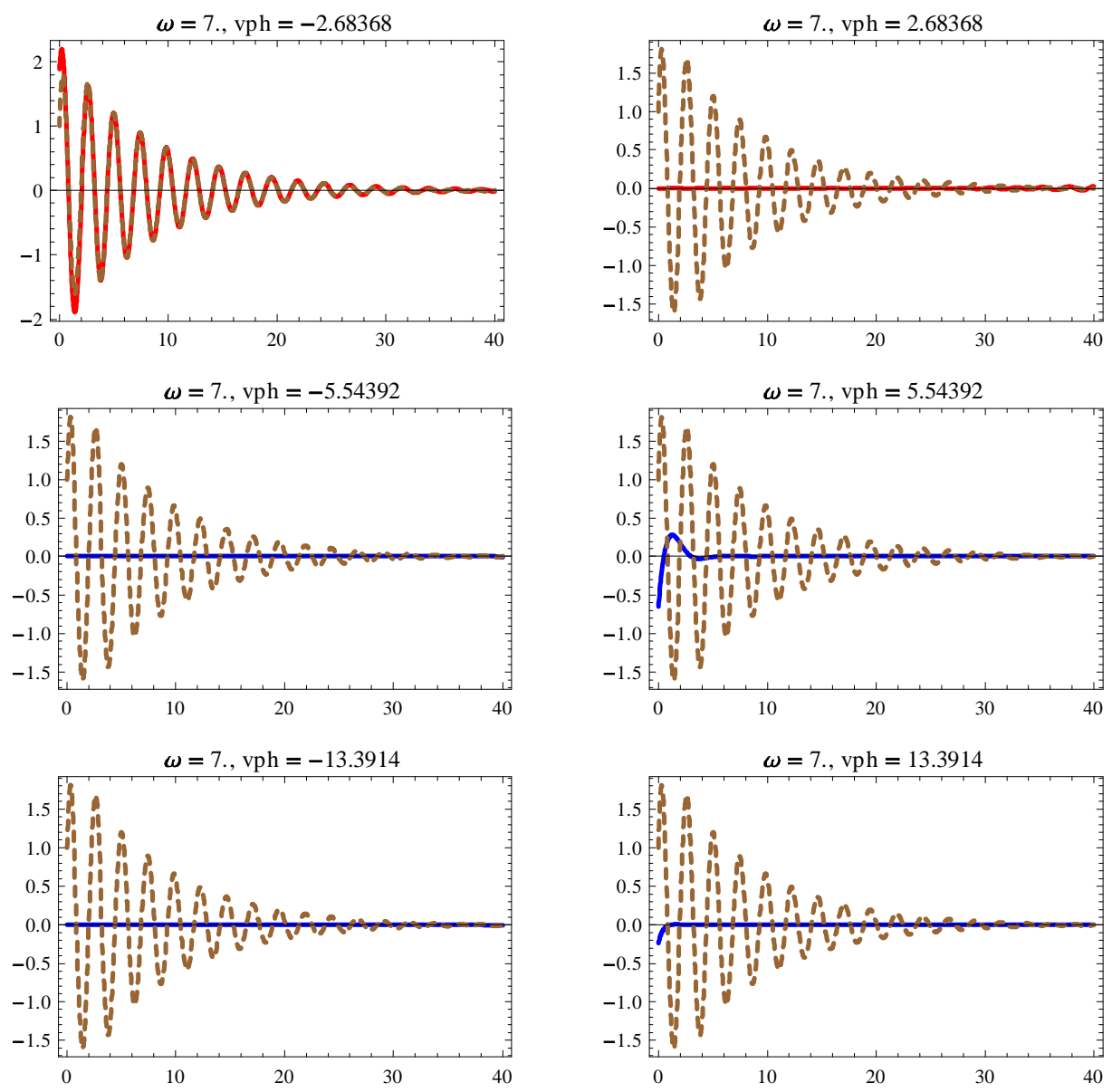

Figure 5: Resonator solution for Burnett equations at $\omega=7, t=0$ : Velocity $v(x, t)$ (dashed, brown) and contributions of the six modes as functions of space. The first two modes are unconventional (red), the other are conventional (blue).

the resonator, due to dissipation in the domain. In short, while $\omega=5$ is a bit large to expect validity of the Burnett equations, they predict reasonable behavior of a damped resonator.

The picture changes drastically as the frequency gets larger. As an example, Fig. 5 shows, for $\omega=7$, overall result (dashed, brown) in comparison to the contribution of the individual modes. As before, the fast conventional modes contribute boundary layer effects at the oscillating boundary. But now the main contribution to the overall result comes from the unconventional mode travelling to the left (red curve in upper left figure, $v_{p h}=-2.684$ ). With this, the overall signal travels towards the moving boundary, with negative signal speed $v_{S}<0$ and negative work $W=-5.02<0$, i.e., this process produces work without a driving force. This solution is a blatant violation of the second law!

\section{Discussion and Conclusions}

Unconventional waves have increasing amplitude in the direction of propagation, while the amplitude of conventional waves decreases in the direction of propagation. 
Models that predict propagation of only unconventional wave type are unphysical in that they give nonsensical results that contradict the second law of thermodynamics, and must be discarded.

Typically, however, models associated with unconventional waves have a larger set of modes, some conventional, some unconventional, with the overall solution a superposition of both. These models must be judged for their overall predictions. The individual waves are just mathematical constructs, that are meaningful only within the superposition, not alone. The fourth order diffusion model considered above provides a simple example of an entropic model - by derivation the second law of thermodynamics is valid for all solutions - that exhibits meaningful solutions as superpositions of conventional and unconventional waves.

The Burnett equations at higher frequencies, on the other hand, provide an example where thermodynamic structure - validity of the second law-breaks down, and the superpositions are dominated by unconventional waves, so that the overall results become nonsensical.

Occurrence of unconventional waves does not suffice to judge negatively on a set of equations. In particular, one has to discard the notion of "spatial instability" promoted in $[6,3]$, since this might lead to dismissal of proper models. If the equations considered, and their boundary conditions, are accompanied by a proper form of the second law of thermodynamics, one will expect sound solutions. If the second law is not available, instead, one has to consider full boundary value problems and evaluate their solutions, which are superpositions of conventional and unconventional waves, with weights that follow from the boundary conditions.

Acknowledgments: Both authors gratefully acknowledge support from the National Science and Engineering Research Council of Canada (NSERC, grant numbers RGPIN-2016-03679 and RGPIN-2018-04573).

\section{A Extended Diffusion Model}

In this Appendix we present some details on the extended diffusion model that is used as one of our examples to study conventional and unconventional wave modes.

\section{A.1 Derivation from 2nd Law}

We denote the deviation of energy (or temperature) from a uniform groundstate as $u(x, t)$, and the heat flux as $q(x, t)$; these, and all other variables and coefficients are dimensionless. We are interested only in a linear theory. The conservation law for energy then reads

$$
\frac{\partial u}{\partial t}+\frac{\partial q}{\partial x}=0
$$

The second law of thermodynamics is used to find a constitutive relation for the heat flux, where we use standard arguments from non-equilibrium thermodynamics [14]. For linear transport laws a quadratic entropy suffices, and we chose a non-equilibrium entropy with gradient contributions,

$$
\eta=\eta_{0}-\frac{1}{2} u^{2}-\frac{\xi}{2}\left(\frac{\partial u}{\partial x}\right)^{2}
$$

where $\eta_{0}$ is the entropy of the equilibrium rest state, and $\xi>0$, so that mathematical entropy $(-\eta)$ is convex [11].

Taking the time derivative of entropy, and repeated use of the conservation law leads to the entropy balance

$$
\frac{\partial \eta}{\partial t}+\frac{\partial \phi}{\partial x}=\sigma
$$


with the entropy flux

$$
\phi=-u q+\xi q \frac{\partial^{2} u}{\partial x^{2}}-\xi \frac{\partial u}{\partial x} \frac{\partial q}{\partial x}
$$

and the entropy generation rate

$$
\sigma=q\left(-\frac{\partial u}{\partial x}+\xi \frac{\partial^{3} u}{\partial x^{3}}\right)
$$

The entropy generation is interpreted as product of the thermodynamic flux $q$ and the thermodynamic force $\left(-\frac{\partial u}{\partial x}+\xi \frac{\partial^{3} u}{\partial x^{3}}\right)$. Entropy generation must be non-negative, $\sigma \geq 0$, and this can be ensured by any linear relation between thermodynamic flux and thermodynamic force with positive phenomenological coefficient. For simplicity, we set the phenomenological coefficient, which for a linear theory is a constant, to unity, and find the heat flux as

$$
q=-\left(\frac{\partial u}{\partial x}-\xi \frac{\partial^{3} u}{\partial x^{3}}\right)
$$

Note, that for $\xi=0$ this model gives the classical diffusion flux, $q=-\frac{\partial u}{\partial x}$.

\section{A.2 Derivation from Microscopic Model}

With the above derivation, we have emphasized the connection between the extended diffusion model and the second law of thermodynamics. To give more meaning to the model, we present an alternative derivation from a kinetic model equation for one-dimensional heat transfer in dimensionless form [18]. The energy transfer is effected by particles that travel with unit velocity in three dimensional space, and carry energy (such as photons or phonons). Thus, on the microscopic level the particles are described through their location in space-time $(x, t)$, and their direction which is expressed through the direction cosine $\cos \vartheta=\mu \in[-1,1]$, where $\vartheta$ is the angle between the particle direction and the $x$-axis. The particle distribution function $f(x, t, \mu)$ is defined such that $f d x d \mu$ gives the energy of particles in the interval $(x, x+d x)$ with direction cosines in $(\mu, \mu+d \mu)$ at time $t$. Thus, the energy density is

$$
\lambda_{0}(x, t)=\int_{-1}^{1} f(x, t, \mu) d \mu .
$$

It is assumed that the particles undergo collisions (among each other, or with a background medium) which only conserve energy; the dimensionless mean free path, i.e. the Knudsen number, is denoted by $\varepsilon$. The kinetic equation for this simple transport model reads

$$
\frac{\partial f}{\partial t}+\mu \frac{\partial f}{\partial x}=-\frac{1}{\varepsilon}\left(f-\frac{1}{2} \lambda_{0}\right)
$$

A hierarchy of moment models can be derived from the kinetic equation by means of Grad's moment method [19]. The discussion in Ref. [18] shows that the required number of moments is related to the desired order in the smallness paramter $\varepsilon$. For rather small $\varepsilon$, a standard diffusion model suffices, but for $\varepsilon>0.1$ (say), Knudsen layer effects at the boundaries arise that can only be approximated when a higher order model is used, with at least 3 variables. The corresponding transport equations read [18]

$$
\begin{aligned}
\frac{\partial \lambda_{0}}{\partial t}+\frac{\partial \lambda_{1}}{\partial x} & =0 \\
\frac{\partial \lambda_{1}}{\partial t}+\frac{1}{3} \frac{\partial \lambda_{0}}{\partial x}+\frac{2}{3} \frac{\partial \lambda_{2}}{\partial x} & =-\frac{1}{\varepsilon} \lambda_{1} \\
\frac{\partial \lambda_{2}}{\partial t}+\frac{2}{5} \frac{\partial \lambda_{1}}{\partial x} & =-\frac{1}{\varepsilon} \lambda_{2}
\end{aligned}
$$


where $\lambda_{n}=\int_{-1}^{1} P_{n}(\mu) f d \mu$ are moments of the distribution function based on Legendre polynomials $P_{n}$.

We proceed with showing the relation between this 3 moment model and the extended diffusion model. For this, we apply the Chapman-Enskog expansion to the moment model $[2,3]$. Energy $\lambda_{0}$ is conserved and hence is not expanded, while the higher order variables are written as a series in the smallness parameter $\varepsilon$,

$$
\begin{aligned}
& \lambda_{1}=\lambda_{1}^{(0)}+\varepsilon \lambda_{1}^{(1)}+\varepsilon^{2} \lambda_{1}^{(2)}+\varepsilon^{3} \lambda_{1}^{(3)} \\
& \lambda_{2}=\lambda_{2}^{(0)}+\varepsilon \lambda_{2}^{(1)}+\varepsilon^{2} \lambda_{2}^{(2)}+\varepsilon^{3} \lambda_{2}^{(3)}
\end{aligned}
$$

This expansion is inserted into the moment equations, and then the expansion coefficients $\lambda_{n}^{(\alpha)}$ are determined successively. For this, it is customary, to replace time derivatives through the conservation law for energy. Proceeding through the orders of $\varepsilon$, one finally finds

$$
\lambda_{1}=-\varepsilon \frac{1}{3} \frac{\partial \lambda_{0}}{\partial x}+\varepsilon^{3} \frac{6}{45} \frac{\partial^{3} \lambda_{0}}{\partial x^{3}} \quad, \quad \lambda_{2}=\varepsilon^{2} \frac{2}{15} \frac{\partial^{3} \lambda_{0}}{\partial x^{3}} .
$$

Note that only the flux $\lambda_{1}$ is required in the conservation law. The second order contribution to $\lambda_{2}$ only plays a role in the expansion, as it leads to a contribution to $\lambda_{1}^{(3)}$. Inserting the third order flux into the conservation law, and rescaling time and space as $t=a \tau, x=b \chi$ with $\frac{a}{3 b^{2}}=1, \frac{2}{5 b^{2}}=\xi$, and setting $\varepsilon=1$ yields the extended diffusion law as presented in Eq. (11),

$$
\frac{\partial \lambda_{0}}{\partial \tau}-\frac{\partial^{2} \lambda_{0}}{\partial \chi^{2}}+\xi \frac{\partial^{4} \lambda_{0}}{\partial \chi^{4}}=0
$$

We note that this derivation of the extended diffusion model is using the same tools as the derivation of the Burnett equations, which result from Chapman-Enskog expansion to 2nd order. While the Burnett equations are unstable, and are not accompanied by a proper formulation of the second law, the diffusion model is stable, and has a proper 2nd law, as was shown in the previous section.

As discussed in detail in Ref. [18], higher order moment models gives rise to Knudsen boundary layers due to the non-equilibrium states induced at boundaries, where particle reflection leads to anisotropic distributions. The first order diffusion model (Fick's law) cannot describe such layers, but higher order moment equations and the extended diffusion model do. These Knudsen layers lead to the differences between classical diffusion model and extended diffusion model in Fig. 2.

\section{A.3 Boundary Conditions}

Boundary conditions must be designed such that the entropy generation at any boundary is positive, and the heat flux is continuous. For simplicity, we assume that the outside material is of the same type but with $\xi=0$, that is classical heat conduction with $q_{B}=-\frac{\partial u_{B}}{\partial x}$ and $\phi_{B}=-u_{B} q_{B}$. The entropy generation at a boundary is, with $q_{B}=q$,

$$
\sigma_{B}=\left(\phi-\phi_{B}\right) \nu=\left[\left(u_{B}-u\right) q+\xi q \frac{\partial^{2} u}{\partial x^{2}}-\xi \frac{\partial u}{\partial x} \frac{\partial q}{\partial x}\right] \nu,
$$

where $\nu$ denotes the normal pointing from the boundary into the domain, i.e., $\nu=+1$ at the left boundary, and $\nu=-1$ at the right boundary.

Inserting the constitutive relation for $q$, this can be brought into the form

$$
\sigma_{B}=\left(-\frac{\partial u}{\partial x} \nu\right)\left[\left(u_{B}-u\right)+\xi^{2} \frac{\partial^{4} u}{\partial x^{4}}\right]+\left(\xi \frac{\partial^{3} u}{\partial x^{3}} \nu\right)\left[\left(u_{B}-u\right)+\xi \frac{\partial^{2} u}{\partial x^{2}}\right]
$$


This interface entropy production can be interpreted as the sum of two products of thermodynamic fluxes (in round brackets) and thermodynamic forces (in square brackets). Boundary conditions that guarantee Onsager symmetry [14] and positive entropy generation are

$$
\text { [Onsager BC] } \quad\left[\begin{array}{c}
-\frac{\partial u}{\partial x} \nu \\
\xi \frac{\partial^{3} u}{\partial x^{3}} \nu
\end{array}\right]=\left[\begin{array}{cc}
\tau_{1} & \tau_{2} \\
\tau_{2} & \tau_{3}
\end{array}\right]\left[\begin{array}{c}
\left(u_{B}-u\right)+\xi^{2} \frac{\partial^{4} u}{\partial x^{4}} \\
\left(u_{B}-u\right)+\xi \frac{\partial^{2} u}{\partial x^{2}}
\end{array}\right],
$$

provided the matrix of coefficients $\tau_{\alpha}$ is semi-positive definite, which requires $\tau_{1} \geq 0$ and $\left(\tau_{1} \tau_{3}-\tau_{2}^{2}\right) \geq 0$. Note that these boundary conditions allow for jumps of $u$ at the boundary.

For an adiabatic boundary, one must set $\tau_{1}=\tau_{2}=\tau_{3}=0$, which guarantees vanishing heat and entropy fluxes, $q=\phi=0$.

\section{References}

[1] D. Burnett, The distribution of molecular velocities and the mean motion in a non-uniform gas. Proc. Lond. Math. Soc. 40, 382-435 (1936)

[2] S. Chapman and T. G. Cowling, The Mathematical Theory of Non-Uniform Gases. Cambridge University Press 1970

[3] H. Struchtrup, Macroscopic Transport Equations for Rarefied Gas Flows-Approximation Methods in Kinetic Theory. Interaction of Mechanics and Mathematics Series, Springer, Heidelberg 2005

[4] A.V. Bobylev, The Chapman-Enskog and Grad methods for solving the Boltzmann equation. Sov. Phys. Dokl. 27, 29-31 (1982)

[5] L.S.García-Colín, R.M.Velasco, and F.J.Uribe, Beyond the Navier-Stokes equations: Burnett hydrodynamics, Physics Reports 465(4), 149-189 (2008)

[6] H. Struchtrup and M. Torrilhon, Regularization of Grad's 13 Moment Equations: Derivation and Linear Analysis. Phys. Fluids 15(9), 2668-2680 (2003)

[7] K.A. Fiscko and D.R. Chapman, Comparison of Burnett, Super-Burnett and Monte Carlo Solutions for Hypersonic Shock Structure. in Proceedings of the 16th Symposium on Rarefied Gasdynamics, 374-395, AIAA, Washington 1989

[8] X. Zhong, R.W. MacCormack, and D.R. Chapman, Stabilization of the Burnett Equations and Applications to High-Altitude Hypersonic Flows. AIAA 91-0770 (1991)

[9] M. Torrilhon and H. Struchtrup, Regularized 13-Moment-Equations: Shock Structure Calculations and Comparison to Burnett Models. J. Fluid Mech. 513, 171-198 (2004)

[10] K.F. Graff, Wave Motion in Elastic Solids. Dover, New York 1975

[11] I. Müller and T. Ruggeri, Rational Extended Thermodynamics. Springer, New York 1998 (Springer Tracts in Natural Philosophy Vol. 37)

[12] M.Sh. Shavaliyev, Super-Burnett Corrections to the Stress Tensor and the Heat Flux in a Gas of Maxwellian Molecules. J. Appl. Maths. Mechs. 57(3), 573-576 (1993) 
[13] R. K. Agarwal, K.Y. Yun, and R. Balakrishnan, Beyond Navier-Stokes: Burnett equations for flows in the continuum-transition regime. Phys. Fluids 13, 3061-3085 (2001), Erratum: Phys. Fluids 14, 1818 (2002)

[14] S.R. De Groot and P. Mazur, Non-equilibrium Thermodynamics. North-Holland, Amsterdam 1969

[15] K.A. Comeaux, D.R. Chapman, and R.W. MacCormack, An Analysis of the Burnett Equations Based on the Second Law of Thermodynamics. AIAA Paper 95-0415, 1995.

[16] H. Struchtrup, Positivity of entropy production and phase density in the Chapman-Enskog expansion. J. of Thermophysics and Heat Transfer 15(3), 372-373 (2001)

[17] H. Struchtrup, Resonance in Rarefied Gases, Cont. Mech. Thermodyn. 24, 361-376 (2012)

[18] H. Struchtrup, Linear Kinetic Heat Transfer: Moment Equations, Boundary Conditions, and Knudsen layers, Physica A 387, 1750-1766 (2008)

[19] H. Grad, On the Kinetic Theory of Rarefied Gases, Comm. Pure Appl. Math. 2, 331-407 (1949) 